\title{
Rhenium Complexes as Highly Active Catalysts for the Hydrosilylation of Carbonyl Compounds
}

\author{
Wenjing Huang§ and Heinz Berke* \\ §SCS Poster Prize Winner
}

\begin{abstract}
Re}\left(\mathrm{P}^{i} \mathrm{Pr}_{3}\right)_{2}(\mathrm{NO})_{2}\right]^{+}\left[\mathrm{B}\left(\mathrm{C}_{6} \mathrm{~F}_{5}\right)_{4}\right]^{-}$(1) and the new NO-functionalized compounds $\left[\operatorname{Re}(\mathrm{H})\left(\mathrm{P}^{i} \mathrm{Pr}_{3}\right)_{2}(\mathrm{NO})(\mathrm{NOB}\right.$ $\left.\left.\left(\mathrm{C}_{6} \mathrm{~F}_{5}\right)_{3}\right)\right](2)$ and $\left[\operatorname{Re}(\mathrm{H})(\mathrm{NO})(\mathrm{NOEt})\left(\mathrm{P}^{i} \mathrm{Pr}_{3}\right)_{2}\right]^{+}\left[\mathrm{B}\left(\mathrm{C}_{6} \mathrm{~F}_{5}\right)_{4}\right]^{-}$(3) were prepared and found to be excellent catalysts for hydrosilylation of ketones. For $\mathbf{2}$ and $\mathbf{3}$ the hydrosilylation procedures are selective, mild, and efficient, and offer very practical methodologies to prepare the respective silyloxy compounds in quantitative yields. In exemplary experiments catalyst 2 could even be recovered.
\end{abstract}

Keywords: Carbonyl compounds · Catalytic hydrosilylation · Coordination chemistry ·

Dinitrosyl rhenium complexes $\cdot \mathrm{NO}$-functionalized compounds

\section{Introduction}

Catalysts play a vital role in industrial processes for fuels, commodity and fine chemicals, pharmaceuticals, and the means for environmental protection. Thus, the development of homogeneous catalysis using transition metal complexes has been one of the more important issues in chemistry in the past decades. However, most of these processes used noble transition metals, which required metal recycling in industrial processes in particular. Rhenium metal is relatively cheap and versatile, but despite this, it has to date only rarely been used in homogeneous or heterogeneous catalysis, in particular it was not used in hydrosilylations. In this paper we describe very efficient non-noble metal hydrosilylation catalysis based on new nitrosyl phosphine rhenium systems.

The unique nitrosyl ligand is one of the strongest $\pi$-acceptors known. It is capable of supporting different oxidation states

${ }^{\star}$ Correspondence: Prof. Dr. H. Berke Universität Zürich

Anorganisch-chemisches Institut

Winterthurerstrasse 190

$\mathrm{CH}-8057$ Zürich

Tel: +4116354680

Fax: +41163568 02

E-Mail: hberke@aci.unizh.ch

http://www.aci.unizh.ch of the metal center and NO coordination modes, and can activate other metal-ligand bonds like the metal-hydrogen bond inducing hydridic polarization and weakening [1]. The NO ligand can formally bind as $\mathrm{NO}^{+}$, causing $\mathrm{M}-\mathrm{N}-\mathrm{O}$ angles of 165 to $180^{\circ}$, or as $\mathrm{NO}^{-}$, with angles between 119 and $140^{\circ}$. A generalized description of the metal-NO interaction was introduced by Feltham and Enemark [2][3], who proposed the $\left\{\mathrm{M}(\mathrm{NO})_{\mathrm{m}}\right\}^{\mathrm{n}}$ formulation, where $\mathrm{n}$ is the sum of the metal d-electrons and the nitrosyl $\pi^{*}$ electrons. For mononitrosyl complexes $\mathrm{MNO}$, the M-N-O angle is predicted to be linear when $n \leq 6$; but could be bent when $n$ $>6$. When $\mathrm{m}>1$, however, the $\left\{\mathrm{M}(\mathrm{NO})_{\mathrm{m}}\right\}^{\mathrm{n}}$ bonding description cannot predict the actual distribution of electrons between the metal and the NO groups, and subsequently also not the $\mathrm{M}-\mathrm{N}-\mathrm{O}$ angle [2].

For catalysis it would be desirable if a linear NO ligand could go bent with little activation energy as predicted for $\mathrm{m}>1$. Adopting the bent $\mathrm{NO}^{-}$state, a free coordination site could thus be opened up.

DFT calculations showed that one of the $\mathrm{NO}$ groups in $\left[\mathrm{Re}(\mathrm{NO})_{2}\left(\mathrm{PR}_{3}\right)_{2} \mathrm{~L}\right]^{\mathrm{n}+}$ complexes $\left(\mathrm{n}=0\right.$ or 1 ; $\mathrm{L}=$ empty site, $\mathrm{H}^{-}, \mathrm{CO}$, $\mathrm{C}_{6} \mathrm{H}_{5} \mathrm{CHO}$, and $\mathrm{ONRe}(\mathrm{NO})\left(\mathrm{PR}_{3}\right)_{2} \mathrm{H} ; \mathrm{R}=$ $\mathrm{Cy}$ or ${ }^{i} \mathrm{Pr}$ ) was bent. The concomitant distortion from a trigonal bipyramid (TBP) to a square pyramid (SP) is dependent on the ease of NO bending, but otherwise dominated by the minimization of steric repulsions in the molecules causing only small energy changes [4].
A series of dinitrosyl rhenium complexes $\left[\mathrm{Re}(\mathrm{H})(\mathrm{NO})_{2}\left(\mathrm{PR}_{3}\right)_{2}\right]\left(\mathrm{R}=\mathrm{Cy},{ }^{i} \mathrm{Pr}\right.$, p-tolyl, $\mathrm{Ph}$ ) [5][6], the corresponding 16electron cations $\left[\operatorname{Re}\left(\mathrm{PR}_{3}\right)_{2}(\mathrm{NO})_{2}\right]^{+}[\mathrm{B}(3,5-$ $\left.\left.\left(\mathrm{CF}_{3}\right)_{2} \mathrm{C}_{6} \mathrm{H}_{3}\right)_{4}\right]^{-}\left(\mathrm{R}=\mathrm{Cy},{ }^{i} \mathrm{Pr}\right)$ [4][7], the dinuclear species $\left[\operatorname{Re}_{2}(H)(\mu, \eta\right.$ $\left.\mathrm{NO})(\mathrm{NO})_{3}\left(\mathrm{PR}_{3}\right)_{4}\right]^{+}\left[\mathrm{B}\left(3,5-\left(\mathrm{CF}_{3}\right)_{2} \mathrm{C}_{6} \mathrm{H}_{3}\right)_{4}\right]^{-}$ $\left(\mathrm{R}=\mathrm{Cy},{ }^{i} \mathrm{Pr}\right)$, [4][7] and the aldehyde complexes $\left[\mathrm{Re}(\mathrm{NO})_{2}(\mathrm{PhCHO})\left(\mathrm{PR}_{3}\right)_{2}\right]^{+}[\mathrm{B}$ $\left.\left(3,5-\left(\mathrm{CF}_{3}\right)_{2} \mathrm{C}_{6} \mathrm{H}_{3}\right)_{4}\right]^{-}\left(\mathrm{R}=\mathrm{Cy},{ }^{i} \mathrm{Pr}\right)$ [4][7] were synthesized earlier in our group. From these, $\left[\operatorname{Re}\left(\mathrm{PR}_{3}\right)_{2}(\mathrm{NO})_{2}\right]^{+}[\mathrm{B}(3,5-$ $\left.\left.\left(\mathrm{CF}_{3}\right)_{2} \mathrm{C}_{6} \mathrm{H}_{3}\right)_{4}\right]^{-} \quad\left(\mathrm{R}=\mathrm{Cy},{ }^{l} \mathrm{Pr}\right)$ and $\left[\mathrm{Re}_{2}(\mathrm{H})(\mu, \eta-\mathrm{NO})(\mathrm{NO})_{3}\left(\mathrm{PR}_{3}\right)_{4}\right]^{+}[\mathrm{B}(3,5-$ $\left.\left.\left(\mathrm{CF}_{3}\right)_{2} \mathrm{C}_{6} \mathrm{H}_{3}\right)_{4}\right]^{-}\left(\mathrm{R}=\mathrm{Cy},{ }^{i} \mathrm{Pr}\right)$ were active in the catalytic scrambling of $\mathrm{H}_{2} / \mathrm{D}_{2}$ to give HD under mild conditions. However, the related hydrogenations and hydrosilylations of $\mathrm{PhCHO}$ gave only low conversions, low TONs and TOFs [8]. One reason for this observation of low activity is connected to the ionic nature of these $\left[\mathrm{B}\left(3,5-\left(\mathrm{CF}_{3}\right)_{2} \mathrm{C}_{6} \mathrm{H}_{3}\right)_{4}\right]^{-}$ salts, which have only low solubility in unpolar solvents, such as toluene or benzene where the catalysis apparently works best. Thus, this series of rhenium salts had first to be anion modified to achieve improved solubilities. In a second step it seemed necessary to also modify the NO ligand to support its bending. For this it offers a Lewis basic site at the oxygen atom. In an earlier study we have for instance reacted the $\left[\mathrm{Re}\left(\mathrm{PR}_{3}\right)_{2}(\mathrm{NO})_{2}\right]^{+}$cation with $\mathrm{HSiEt}_{3}$ to obtain the $\mathrm{SiEt}_{3}{ }^{+}$adduct $\left[\mathrm{Re}(\mathrm{H})\left(\left(\mathrm{PR}_{3}\right)_{2}(\mathrm{~N}\right.\right.$ $\left.\mathrm{O})\left(\mathrm{NOSiEt}_{3}\right)\right]^{+}\left[\mathrm{B}\left(3,5-\left(\mathrm{CF}_{3}\right)_{2} \mathrm{C}_{6} \mathrm{H}_{3}\right)_{4}\right]^{-}(\mathrm{R}=$ $\mathrm{Cy}$ or ${ }^{i} \mathrm{Pr}$ ) [7] by heterolysis of the silane. 
In the present work rhenium dinitrosyl cations and the parent hydride systems were therefore modified by either anion replacement with $\left[\mathrm{B}\left(\mathrm{C}_{6} \mathrm{~F}_{5}\right)_{4}\right]^{-}$or by attachment of the electrophiles $\mathrm{B}\left(\mathrm{C}_{6} \mathrm{~F}_{5}\right)_{3}$ and $\mathrm{Et}^{+}$to one of the NO groups.

\section{Results and Discussions}

Hydrosilylation is often used as a convenient alternative to hydrogenation. According to Scheme 1 catalytic hydrosilylations of a variety of carbonyl compounds were carried out with the catalysts $\left[\operatorname{Re}\left(\mathrm{P}^{i} \mathrm{Pr}_{3}\right)_{2}\right.$ $\left.(\mathrm{NO})_{2}\right]^{+}\left[\mathrm{B}\left(\mathrm{C}_{6} \mathrm{~F}_{5}\right)_{4}\right]^{-}(\mathbf{1}),\left[\operatorname{Re}(\mathrm{H})\left(\mathrm{P}^{i} \mathrm{Pr}_{3}\right)_{2}(\mathrm{~N}\right.$ $\left.\mathrm{O})\left(\mathrm{NOB}\left(\mathrm{C}_{6} \mathrm{~F}_{5}\right)_{3}\right)\right](2)$, and $\left[\mathrm{Re}(\mathrm{H})\left(\mathrm{P}^{i} \mathrm{Pr}_{3}\right)_{2}\right.$ (NO)(NOEt) $]^{+}\left[\mathrm{B}\left(\mathrm{C}_{6} \mathrm{~F}_{5}\right)_{4}\right]^{-}(\mathbf{3})$ and $\mathrm{HSiR}_{3}^{3}$ $\left(\mathrm{R}^{3}=\mathrm{Et}\right.$ or $\left.\mathrm{Ph}\right)$ at various temperatures. Either toluene or no solvent was applied as indicated in the Table. The products were silylated alcohols, which were analyzed by ${ }^{1} \mathrm{H}$ and ${ }^{13} \mathrm{C}\left\{{ }^{1} \mathrm{H}\right\}$ NMR spectroscopy and GC-MS.

For the hydrosilylations the most remarkable observations were:

1) The hydrosilylation procedure was clean, mild and efficient and gave quantitative yields in the organic products. Thus, these catalytic reactions offer a very practical and general method to prepare silyl alcohols;

2) TONs up to 9000 and TOFs up to 22500 $\mathrm{h}^{-1}$ were observed. High activities and regioselectivities were accomplished;

3) The hydrosilylation of ketones can be advantageously conducted in neat ketone liquids without addition of solvents. The catalyst precipitated at the end of the reaction and could be recycled at least three times. These observations bear a close resemblance with Bullock's recent results [9].

Based on several observations a mechanistic scheme for the hydrosilylation reaction is proposed. It comprises a $\mathrm{NO}$ bent species A with a vacant coordination site, which gets further support in $\mathbf{2}$ and $\mathbf{3}$ from the Lewis acid interaction. When 2 was mixed with 1-hexene in toluene- $\mathrm{d}_{8}$ isomerization to 2-hexene was observed. This indicates that an insertion/deinsertion equilibrium similar to the step $\mathbf{B} \leftrightarrows \mathbf{C}$ of Scheme 2 is in operation.

Based on the observation of $\mathrm{H}_{2} / \mathrm{D}_{2}$ scrambling to afford HD using the same complexes, species $\mathbf{C}$ is anticipated to reversibly take up $\mathrm{H}_{2}$ or $\mathrm{D}_{2}$ to give a dihydrogen/dideuterium hydride complex, which would undergo $\mathrm{H} / \mathrm{D}$ exchange by $\mathrm{H}_{2}$ or $\mathrm{D}_{2}$ oxidative addition to form an isotopomeric mixture of a rhenium(+III) trihydride species<smiles>[R]C([R])=O</smiles>
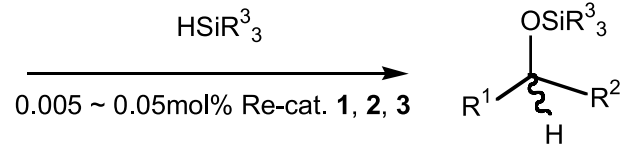

$\mathrm{R}^{1}, \mathrm{R}^{2}=\mathrm{H}$, Alkyl, Aryl; $\mathrm{R}^{3}=\mathrm{Ph}$, Et; r.t. to $70^{\circ} \mathrm{C}$; toluene or without solvent

Scheme 1.

Table. Various hydrosilylations of carbonyl compounds with the isopropylphosphine substituted rhenium catalysts $1-3$ and $\mathrm{R}_{3}^{3} \mathrm{SiH}\left(\mathrm{R}^{3}=\mathrm{Et}, \mathrm{Ph}\right)$

\begin{tabular}{|c|c|c|c|c|c|c|c|c|}
\hline \multirow{2}{*}{ Entry } & \multicolumn{2}{|c|}{ Substrate (S) } & \multirow[t]{2}{*}{ Re-cat (C) } & \multirow[t]{2}{*}{$\mathrm{S}: \mathrm{C}$} & \multirow[t]{2}{*}{ Temp $\left[{ }^{\circ} \mathrm{C}\right]$} & \multirow[t]{2}{*}{ solvent } & \multirow[t]{2}{*}{ Yielda [\%] } & \multirow[t]{2}{*}{$\mathrm{TOF}\left[\mathrm{h}^{-1}\right]$} \\
\hline & $\mathrm{R}^{1} \mathrm{R}^{2} \mathrm{CO}$ & $\mathrm{R}^{3}$ & & & & & & \\
\hline 1 & $\mathrm{PhCHO}$ & Et & 1 & 100 & r.t. & toluene & 48 & n. d. \\
\hline 2 & PhCOMe & $\mathrm{Ph}$ & 1 & 250 & 70 & toluene & 61 & n. d. \\
\hline 3 & PhCOMe & $\mathrm{Ph}$ & 2 & 2000 & 40 & toluene & 100 & 2820 \\
\hline 4 & MeCOEt & $\mathrm{Ph}$ & $2^{b}$ & 1600 & 40 & toluene & 100 & 22500 \\
\hline 5 & $\mathrm{PhCOPh}$ & $\mathrm{Ph}$ & 2 & 2000 & 70 & toluene & 100 & 1290 \\
\hline 6 & $\mathrm{PhCHO}$ & Et & 2 & 2000 & r.t. & neat & 100 & c \\
\hline 7 & $\mathrm{PhCHO}$ & $\mathrm{Ph}$ & 2 & 10000 & 70 & toluene & 93 & n. d. \\
\hline 8 & MeCOMe & Et & 3 & 2000 & r.t. & neat & 96 & n. d. \\
\hline
\end{tabular}

$\mathrm{S}: \mathrm{C}=$ Substrate/catalyst molar ratios; Carbonyl compounds: $\mathrm{R}_{3}{ }_{3} \mathrm{SiH}=1: 1$; r.t.: room temperature; neat: without solvent; $n$. d.: not determined; Re-cat. : 1, 2, and 3; a Yields determined by ${ }^{1} \mathrm{H}$ NMR spectroscopy; ${ }^{b} \mathrm{Re}$-cat: here $\left[\mathrm{Re}\left(\mathrm{PC} \mathrm{y}_{3}\right)_{2}(\mathrm{NO})_{2}\right]^{+}\left[\mathrm{B}\left(\mathrm{C}_{6} \mathrm{~F}_{5}\right)_{4}\right]^{-;}$; reaction finished in a few seconds; it is faster than that of entry 4 , TOF not determined. or would directly undergo scrambling by $\sigma$ bond metathesis (SMB). The oxidative addition/reductive elimination sequence or SMB would both provide possible pathways for the formation and elimination of the silylated ketone products from $\mathbf{D}$. If the hydrosilylation catalyses are carried out with complexes $\mathbf{1}$, heterolytic splitting of one equivalent of $\mathrm{R}_{3}^{3} \mathrm{SiH}\left(\mathrm{R}^{3}=\mathrm{Et}\right.$ or $\left.\mathrm{Ph}\right)$ would be a preceding step to the catalytic cycle. $\left[\operatorname{Re}(\mathrm{H})\left(\left(\mathrm{PR}_{3}\right)_{2}\right.\right.$ $\left.(\mathrm{NO})\left(\mathrm{NOSiR}_{3}\right)\right]^{+}$complexes are anticipated to be formed, which could drive the catalytic cycle in a way related to $\mathbf{2}$ and $\mathbf{3}$.

\section{Experimental}

Unless specified, all manipulations with air-sensitive compounds and involving organometallic complexes were performed in oven-dried glassware using a combination of nitrogen-filled Vacuum Atmospheres glovebox, high vacuum, and Schlenk techniques under a dry nitrogen atmosphere. NMR spectra were obtained on a Varian Gemini-200 or Varian Gemini-300 spectrometer at ambient temperature, if not otherwise stated. IR spectra were recorded on a Biorad FTS-45 spectrophotometer. Raman spectra were recorded on a Renishaw Labram Raman spectrometer. GC-MS spectra were recorded on a Varian CP-3800 instrument. Elemental analyses were performed on a Leco $\mathrm{CHN}(\mathrm{S})-932$ instrument at the University of Zürich.

The complexes $\left[\mathrm{Re}(\mathrm{H})\left(\mathrm{P}^{i} \mathrm{Pr}_{3}\right)_{2}(\mathrm{NO})_{2}\right]$ [5], $\quad \mathrm{B}\left(\mathrm{C}_{6} \mathrm{~F}_{5}\right)_{3} \quad$ [10] and $\left[\mathrm{H}\left(\mathrm{OEt}_{2}\right)_{2}\right]^{+}\left[\mathrm{B}\left(\mathrm{C}_{6} \mathrm{~F}_{5}\right)_{4}\right]^{-} \quad[11]$ were prepared according to reported procedures. $\left[\mathrm{OEt}_{3}\right]^{+}\left[\mathrm{B}\left(\mathrm{C}_{6} \mathrm{~F}_{5}\right)_{4}\right]^{-}$was prepared by ion metathesis of $\left[\mathrm{OEt}_{3}\right]^{+}\left[\mathrm{BF}_{4}\right]^{-}$with $\mathrm{Li}^{+}\left[\mathrm{B}\left(\mathrm{C}_{6} \mathrm{~F}_{5}\right)_{4}\right]^{-}$in ether. Solvents were dried by standard methods and freshly distilled under nitrogen before use (e.g. toluene, pentane, and diethylether were distilled over metal sodium/benzophenone; dichloromethane was freshly distilled over $\mathrm{P}_{2} \mathrm{O}_{5}$, then over $\mathrm{CaH}_{2}$ ). The deuterated solvents were also dried over appropriate drying agents, three freeze-pump-thaw cycles prior to degassing, and were then transferred to a vacuum line before use. Organic unsaturated compounds were obtained from Aldrich; $\mathrm{Ph}_{3} \mathrm{SiH}, \mathrm{Et}_{3} \mathrm{SiH}$ were obtained from Fluka; all were used as received

\section{Catalytic Hydrosilylations}

A typical hydrosilylation was carried out as follows: The carbonyl compound and the respective silane were put in a $5 \mathrm{~mm}$ Young NMR tube together with toluene$\mathrm{d}_{8}$ (1:1 ratio). The catalyst was placed on the top of the tube avoiding early mixing with the organic sample. After freezing the sample by liquid nitrogen, the catalyst was brought down to the surface of the organic 


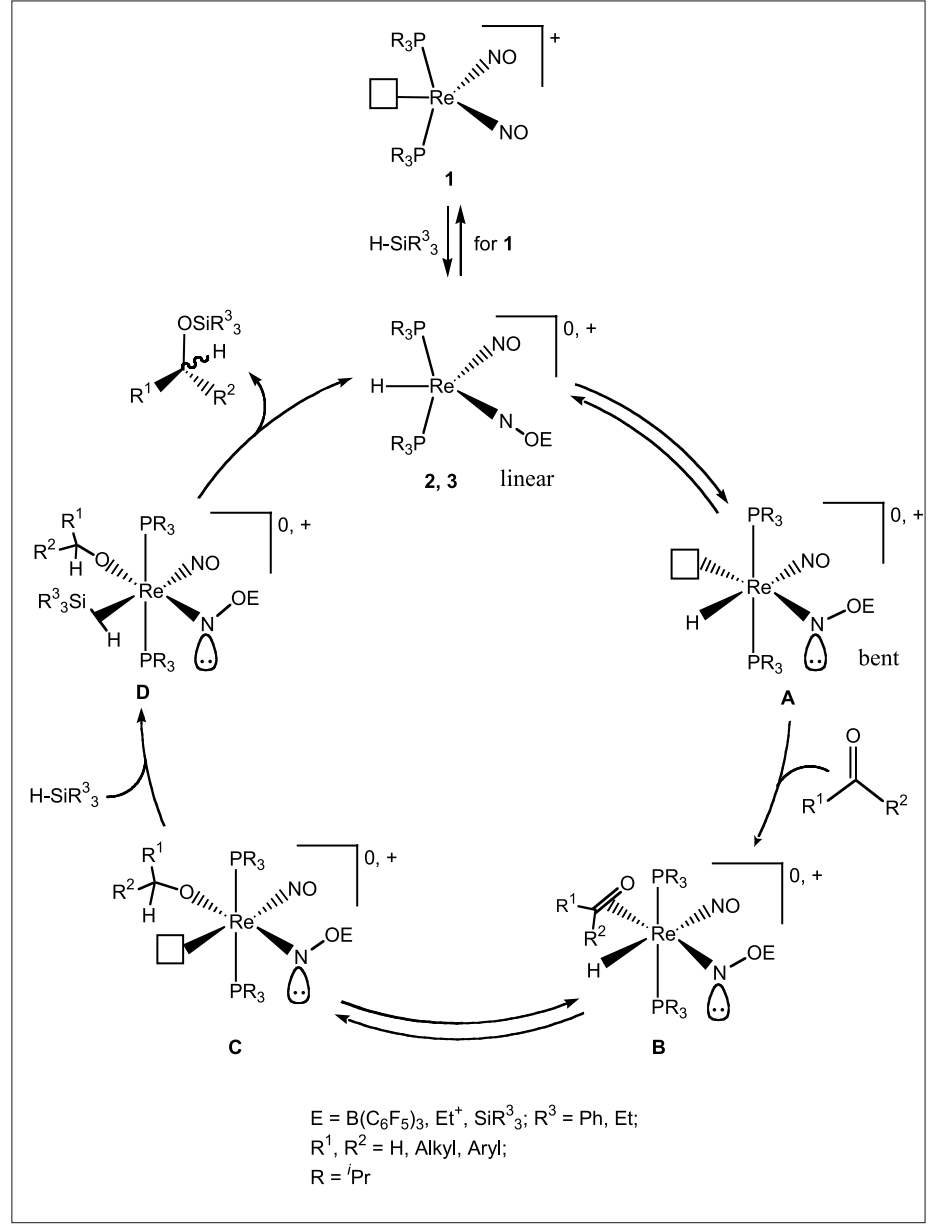

Scheme 2

sample. Reactions were followed by ${ }^{1} \mathrm{H}$ and ${ }^{13} \mathrm{C}\left\{{ }^{1} \mathrm{H}\right\}$ NMR spectroscopy at the required temperature. An elapsed time of 3 min was allowed before measurements were started. The catalyst was filtered off through a column of silica gel and evaporation of the solvent afforded the products, which were analyzed by ${ }^{1} \mathrm{H}$ and ${ }^{13} \mathrm{C}\left\{{ }^{1} \mathrm{H}\right\}$ NMR spectroscopy and GC-MS.

\section{Preparations of the Complexes $\left[\operatorname{Re}\left(\mathbf{P}^{i} \mathrm{Pr}_{3}\right)_{2}(\mathrm{NO})_{2}\right]^{+}\left[\mathrm{B}\left(\mathrm{C}_{6} \mathbf{F}_{5}\right)_{4}\right]^{-}(\mathbf{1})$}

A heterogeneous mixture containing $\left[\operatorname{Re}(\mathrm{H})\left(\mathrm{P}^{i} \mathrm{Pr}_{3}\right)_{2}(\mathrm{NO})_{2}\right](56.8 \mathrm{mg}, 0.1 \mathrm{mmol})$ and $\left[\mathrm{H}\left(\mathrm{OEt}_{2}\right)_{2}\right]^{+}\left[\mathrm{B}\left(\mathrm{C}_{6} \mathrm{~F}_{5}\right)_{4}\right]^{-}(91.2 \mathrm{mg}, 0.11$ $\mathrm{mmol}$ ) in toluene ( $3 \mathrm{ml}$ ) was stirred for $2 \mathrm{~h}$. Immediate evolution of $\mathrm{H}_{2}$ takes place. The volume was reduced to $0.5 \mathrm{ml}$ under vacuum, and then $5 \mathrm{ml}$ cooled pentane $\left(-30{ }^{\circ} \mathrm{C}\right)$ was added. The supernatant liquid was removed and the oily residue dried under vacuum. The red residue as washed with toluene/pentane (1:10) $(2 \times 5$ l) and dried under vacuum, leaving $114.8 \mathrm{mg}$ of $\mathbf{1}(92 \%)$. Anal. calcd. for $\mathrm{C}_{42} \mathrm{H}_{42} \mathrm{BF}_{20} \mathrm{~N}_{2} \mathrm{O}_{2} \mathrm{P}_{2}$ Re: C 40.49; H 3.40; N 2.25. Found: C 40.52; H 3.75; N 2.65. IR (cm ${ }^{-1}$, solid, ATR): 1709, 1648. ${ }^{1} \mathrm{H}$ NMR (300.1 MHz, toluene-d $\left.{ }_{8}\right): \delta 1.04$ (m, $\left[\mathrm{P}(\mathrm{CHMe})_{3}\right]_{2}, 2.32$ (m, $\left.\mathrm{P}(\mathrm{CHMe})_{3}\right)$. ${ }^{31} \mathrm{P}\left\{{ }^{1} \mathrm{H}\right\}$ NMR $\left(121.5 \mathrm{MHz}\right.$, toluene-d $\left.\mathrm{d}_{8}\right): \delta$ 57.3 (s, br, $\left.P\left(\mathrm{CHMe}_{2}\right)_{3}\right) .{ }^{19} \mathrm{~F}$ NMR $(282.3$ $\mathrm{MHz}$, toluene- $\left.\mathrm{d}_{8}\right): \delta-167.7\left(\mathrm{t}, 8 \mathrm{~F},{ }^{3} J_{\mathrm{FF}}=\right.$ $\left.18 \mathrm{~Hz}, m-\mathrm{C}_{6} \mathrm{~F}_{5}\right),-164.1\left(\mathrm{t}, 4 \mathrm{~F},{ }^{3} J_{\mathrm{FF}}=20.9\right.$
$\left.\mathrm{Hz}, p-\mathrm{C}_{6} \mathrm{~F}_{5}\right),-132.6\left(\mathrm{~d}, 8 \mathrm{~F},{ }^{3} J_{\mathrm{FF}}=19.8 \mathrm{~Hz}\right.$, $\left.o-\mathrm{C}_{6} \mathrm{~F}_{5}\right)$.

$\left[\operatorname{Re}(\mathrm{H})\left(\mathbf{P}^{i} \mathbf{P r}_{3}\right)_{2}(\mathrm{NO})\left(\mathrm{NOB}\left(\mathrm{C}_{6} \mathbf{F}_{5}\right)_{3}\right)\right](2)$

$\left[\operatorname{Re}(\mathrm{H})\left(\mathrm{P}^{i} \mathrm{Pr}_{3}\right)_{2}(\mathrm{NO})_{2}\right](56.8 \mathrm{mg}, 0.1 \mathrm{mmol})$ and $\mathrm{B}\left(\mathrm{C}_{6} \mathrm{~F}_{5}\right)_{3}(51.2 \mathrm{mg}, 0.1 \mathrm{mmol})$ were stirred for $0.5 \mathrm{~h}$ in toluene $(3 \mathrm{ml})$. After this time the yellow solution was filtered off and dried under vacuum to yield $105.8 \mathrm{mg}$ $(98 \%)$ of a yellow powder of 2 . Anal. calcd. for $\mathrm{C}_{36} \mathrm{H}_{43} \mathrm{BF}_{15} \mathrm{~N}_{2} \mathrm{O}_{2} \mathrm{P}_{2} \mathrm{Re}$ : $\mathrm{C} 40.05 ; \mathrm{H} 4.01$; $\mathrm{N}$ 2.59. Found: C 40.17; H 3.85; N 2.63. IR ( $\mathrm{cm}^{-1}$, solid, ATR): $1943(\mathrm{Re}-\mathrm{H}), 1641$ (NO), 1340 (N-OB). Raman ( $\mathrm{cm}^{-1}$, solid): 1938 (Re-H), 1649 (NO), 1343 (N-OB). ${ }^{1} \mathrm{H}$ NMR (300.1 MHz, toluene- $\left.\mathrm{d}_{8}\right): \delta 0.77(\mathrm{~m}$, $\left.\mathrm{P}(\mathrm{CHMe})_{3}\right), 1.79\left(\mathrm{~m}, \mathrm{P}\left(\mathrm{CHMe}_{2}\right)_{3}\right), 4.07$ $\left(\mathrm{t}, 1 \mathrm{H},{ }^{2} J_{\mathrm{HP}}=40.7 \mathrm{~Hz}, \operatorname{Re}-H\right) .{ }^{31} \mathrm{P}\left\{{ }^{1} \mathrm{H}\right\}$ NMR (121.5 MHz, toluene- $\left.\mathrm{d}_{8}\right)$ : $\delta 53.5$ $\left(\mathrm{s}, P\left(\mathrm{CHMe}_{2}\right)_{3}\right) \cdot{ }^{19} \mathrm{~F}$ NMR $(282.3 \mathrm{MHz}$, toluene- $\mathrm{d}_{8}$ ): $\delta-165.8$ (pseudo t, 6F, ${ }^{3} J_{\mathrm{FF}}$ $\left.=18.7 \mathrm{~Hz}, m-\mathrm{C}_{6} \mathrm{~F}_{5}\right),-159.6\left(\mathrm{t}, 3 \mathrm{~F},{ }^{3} J_{\mathrm{FF}}\right.$ $\left.=19.8 \mathrm{~Hz}, p-\mathrm{C}_{6} \mathrm{~F}_{5}\right),-131.3\left(\mathrm{~d}, 6 \mathrm{~F},{ }^{3} J_{\mathrm{FF}}=\right.$ $\left.20.9 \mathrm{~Hz}, o-\mathrm{C}_{6} \mathrm{~F}_{5}\right) .{ }^{13} \mathrm{C}\left\{{ }^{1} \mathrm{H}\right\} \mathrm{NMR}(75.5 \mathrm{~Hz}$, toluene- $\left.\mathrm{d}_{8}\right): \delta 18.9\left(\mathrm{~s}, \mathrm{P}(\mathrm{CHMe})_{3}\right), 19.4$ $\left(\mathrm{s}, \mathrm{P}(\mathrm{CHMe})_{3}\right), 28.4$ (pseudo t, ${ }^{1} J_{\mathrm{CP}}=14.4$ $\mathrm{Hz}$, ipso $\left.\mathrm{P}\left(\mathrm{C} \mathrm{HMe}_{2}\right)_{3}\right), \sim 120\left(\mathrm{~m}\right.$, ipso- $\left.\mathrm{C}_{6} \mathrm{~F}_{5}\right)$, $140.4\left(\mathrm{dm},{ }^{1} J_{\mathrm{CF}}=235.4 \mathrm{~Hz}, p-\mathrm{C}_{6} \mathrm{~F}_{5}\right), 137.7$ $\left(\mathrm{dm},{ }^{1} J_{\mathrm{CF}}=243.9 \mathrm{~Hz}, m-\mathrm{C}_{6} \mathrm{~F}_{5}\right), 148.8(\mathrm{dm}$, $\left.{ }^{1} J_{\mathrm{CF}}=241.8 \mathrm{~Hz}, o-\mathrm{C}_{6} \mathrm{~F}_{5}\right)$.

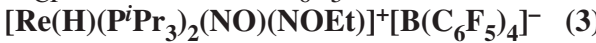
$\left[\mathrm{Re}(\mathrm{H})\left(\mathrm{PiPr}_{3}\right)_{2}(\mathrm{NO})_{2}\right](56.8 \mathrm{mg}, 0.1 \mathrm{mmol})$ and $\left[\mathrm{OEt}_{3}\right]^{+}\left[\mathrm{B}\left(\mathrm{C}_{6} \mathrm{~F}_{5}\right)_{4}\right]^{-}(79.0 \mathrm{mg}, \quad 0.1$ $\mathrm{mmol})$ in ether $(3 \mathrm{ml})$ was stirred for $3 \mathrm{~h}$.
The yellow solution was filtered off and dried under vacuum, then the yellow powder was washed with ether/pentane (1:10) $(3 \mathrm{x} 10 \mathrm{ml})$ and dried under vacuum, to yield $118.2 \mathrm{mg}(93 \%)$ of $\mathbf{3}$ as a yellow powder. Anal. calcd. for $\mathrm{C}_{44} \mathrm{H}_{48} \mathrm{BF}_{20} \mathrm{~N}_{2} \mathrm{O}_{2} \mathrm{P}_{2} \mathrm{Re}$ : C 41.42; H 3.79; N 2.20. Found: C 41.75; $\mathrm{H}$ 3.43; N 2.28. IR ( $\mathrm{cm}^{-1}$, solid, ATR): 1640 (NO), 1372 (N-OEt), 1956 (Re-H). Raman $\left(\mathrm{cm}^{-1}\right.$, solid): 1641 (NO), $1374(\mathrm{~N}-$ OEt), 1958 (Re-H). ${ }^{1} \mathrm{H}$ NMR (300.1 MHz, THF-d $\left._{8}\right): \delta 1.36\left(\mathrm{~m}, \mathrm{P}\left(\mathrm{CHM} e_{2}\right)_{3}\right), 1.44$ $\left(\mathrm{t},{ }^{2} J_{\mathrm{HH}}=7.2 \mathrm{~Hz}, \mathrm{NOCH}_{2} \mathrm{CH}_{3}\right), 2.71(\mathrm{~m}$, $\left.\mathrm{P}\left(\mathrm{CHMe}_{2}\right)_{3}\right), 4.77$ (quart, ${ }^{2} J_{\mathrm{HH}}=7.2 \mathrm{~Hz}$, $\left.\mathrm{NOCH}_{2} \mathrm{CH}_{3}\right), 5.50\left(\mathrm{t},{ }^{2} \mathrm{~J}_{\mathrm{HP}}=38.3 \mathrm{~Hz}, \mathrm{Re}-\right.$ H). ${ }^{31} \mathrm{P}\left\{{ }^{1} \mathrm{H}\right\}$ NMR $\left(121.5 \mathrm{MHz}, \mathrm{THF}-\mathrm{d}_{8}\right)$ : $\delta 59.2\left(\mathrm{~s}, P\left(\mathrm{CHMe}_{2}\right)_{3}\right) \cdot{ }^{19} \mathrm{~F}$ NMR $(282.3$

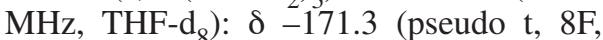
$\left.{ }^{3} J_{\mathrm{FF}}=18 \mathrm{~Hz}, m-\mathrm{C}_{6} \mathrm{~F}_{5}\right),-167.8\left(\mathrm{t}, 4 \mathrm{~F},{ }^{3} J_{\mathrm{FF}}=\right.$ $\left.20.6 \mathrm{~Hz}, p-\mathrm{C}_{6} \mathrm{~F}_{5}\right),-135.1\left(\mathrm{~d}, 8 \mathrm{~F},{ }^{3} J_{\mathrm{FF}}=19.8\right.$ $\left.\mathrm{Hz}, o-\mathrm{C}_{6} \mathrm{~F}_{5}\right) .{ }^{13} \mathrm{C}\left\{{ }^{1} \mathrm{H}\right\}$ NMR $(75.5 \mathrm{MHz}$, THF-d ${ }_{8}$ ): $\delta 13.0\left(\mathrm{~s}, \mathrm{NOCH}_{2} \mathrm{CH}_{3}\right), 19.5$ (s, $\left.\mathrm{P}(\mathrm{CHMe})_{3}\right), 19.7$ (s, $\left.\mathrm{P}(\mathrm{CHMe})_{3}\right), 28.2$ (pseudo t, ${ }^{1} J_{\mathrm{CP}}=14.6 \mathrm{~Hz}$, ipso $\mathrm{P}\left(\mathrm{CHMe}_{2}\right)_{3}$ ), $81.0\left(\mathrm{~s}, \mathrm{NOCH}_{2} \mathrm{CH}_{3}\right)$.

\section{Acknowledgements}

Financial support from the Swiss National ScienceFoundation and the funds at the University of Zurich are gratefully acknowledged.

Received: January 12, 2005

[1] H. Berke, P. Burger, Comments on Inorganic Chemistry 1994, 16, 279; B.E. Bursten, M.G. Gatter, J. Am. Chem. Soc. 1984, 106, 2554; H. Jacobsen, H. Berke, in 'Recent Advances in Hydride Chemistry', 1st ed., Eds. M. Peruzzi, R. Poli, Elsevier, New York, 2001.

[2] R.D. Feltham, J.H. Enemark, Top. Stereochem. 1981, 12, 155.

[3] J.H. Enemark, R.D. Feltham, Coord. Chem. Rev. 1974, 13, 339; J. H. Enemark, R. D. Feltham, J. Am. Chem. Soc. 1974, 96, 5002

[4] H. Jacobsen, K. Heinze, A. Llamazares, H.W. Schmalle, G. Artus, H. Berke, J. Chem. Soc. Dalton Trans. 1999, 1717.

[5] D. Gusev, A. Llamazares, G. Artus, H. Jacobsen, H. Berke, Organometallics 1999, 18,75 .

[6] $\mathrm{R}=p$-tolyl; W. Huang, H. Berke, unpublished results.

[7] A. Llamazares, H.W. Schmalle, H. Berke, Organometallics 2001, 20, 5277.

[8] A. Llamazares, H. Berke, unpublished results.

[9] V.K. Dioumaev, R.M. Bullock, Nature 2003, 424, 530.

[10] A.N. Chernega, A.J. Graham, M.L.H. Green, J. Haggitt, J. Lloyd, C.P. Mehnert, N. Metzler, J. Souter, J. Chem. Soc. Dalton Trans. 1997, 2293.

[11] P. Jutzi, C. Muller, A. Stammler, H.G. Stammler, Organometallics 2000, 19 1442. 